\title{
Skeletal muscle morphology in sarcopenia defined using the EWGSOP criteria: findings from the Hertfordshire Sarcopenia Study (HSS)
}

\author{
H. P. Patel ${ }^{1,2,3^{*}}$, M. C. White ${ }^{1}$, L. Westbury ${ }^{2}$, H. E. Syddall², P. J. Stephens ${ }^{1}$, G. F. Clough ${ }^{4}$, C. Cooper ${ }^{2,3,6}$ and A. A. Sayer ${ }^{1,2,3,5,7}$
}

\begin{abstract}
Background: Sarcopenia is defined as the loss of muscle mass and function with age and is associated with decline in mobility, frailty, falls and mortality. There is considerable interest in understanding the underlying mechanisms. Our aim was to characterise muscle morphology changes associated with sarcopenia among community dwelling older men.

Methods: One hundred and five men aged 68-76 years were recruited to the Hertfordshire Sarcopenia Study (HSS) for detailed characterisation of muscle including measures of muscle mass, strength and function. Muscle tissue was obtained from a biopsy of the vastus lateralis for 99 men and was processed for immunohistochemical studies to determine myofibre distribution and area, capillarisation and satellite cell (SC) density.

Results: Six (6\%) men had sarcopenia as defined by the European Working Group on Sarcopenia in Older People (EWGSOP) criteria. These men had lower SC density ( 1.7 cells $/ \mathrm{mm}^{2}$ vs 3.8 cells $\left./ \mathrm{mm}^{2}, p=0.06\right)$ and lower SC/fibre ratio $(0.02$ vs $0.06, p=0.06)$ than men without sarcopenia. Although men with sarcopenia tended to have smaller myofibres and lower capillary to fibre ratio, these relationships were not statistically significant.

Conclusion: We have shown that there may be altered muscle morphology parameters in older men with sarcopenia. These results have the potential to help identify cell and molecular targets for therapeutic intervention. This work now requires extension to larger studies which also include women.
\end{abstract}

Keywords: Sarcopenia, Muscle morphology, Community dwelling older men

\section{Background}

Sarcopenia is associated with adverse health outcomes and incurs a substantial health care cost [1-3]. Defined as the loss of skeletal muscle mass and function, sarcopenia is common in both men and women over the age of 65 across a range of healthcare settings [4]. For example, among community dwelling older people in the UK, prevalence rates for sarcopenia have been estimated at $4.6 \%$ for men and $7.9 \%$ for women [5]. Sarcopenia has been defined based on lean mass indices i.e., total lean

\footnotetext{
* Correspondence: hp@mrc.soton.ac.uk

'Academic Geriatric Medicine, University of Southampton, University Hospital Southampton FoundationTrust (UHSFT), Tremona Road, Southampton SO16 6YD, UK

${ }^{2}$ Medical Research Council Lifecourse Epidemiology Unit, University of Southampton, UHSFT, Tremona Road, Southampton SO16 6YD, UK Full list of author information is available at the end of the article
}

mass, appendicular lean mass and muscle function i.e., grip strength or physical performance. Notable diagnostic algorithms include The European Working Group on Sarcopenia in Older People (EWGSOP) [6], The Foundation for the National Health Institutes of Health (FNIH) Sarcopenia Project [7], and the Asian Working Group for Sarcopenia (AWGS) [8], the latter definition to account for ethnic variations in muscle mass and muscle function.

There is considerable interest in understanding the mechanisms driving sarcopenia and there have been a number of small studies investigating morphological changes in skeletal muscle with increasing age. For example, it has been reported that between the sixth and ninth decades, myofibre size and number decrease in both men and women [9]. In addition there is type I and type II myofibre atrophy, as well as other changes in 
muscle morphology including the appearance of hybrid fibres, the presence of hypertrophied fibres and fibre type grouping [10-12]. Although not completely understood, several factors contribute to these morphological changes including, inflammation, denervation, oxidative stress, imbalance in protein synthesis and reduced satellite cell number and or function [13].

Studies of muscle morphology have rarely been population based and how these morphological changes relate to altered skeletal muscle mass, function and sarcopenia is unclear. The objective of this study was therefore to determine the relationship between muscle morphology and sarcopenia as defined using the European Working Group Sarcopenia in Older People (EWGSOP) criteria in a population based study of community dwelling older men.

\section{Methods}

\section{Study participants}

One hundred and five community dwelling older men aged 68-76 years who had participated in the UK Hertfordshire Cohort Study (HCS) [14] were involved in the Hertfordshire Sarcopenia Study (HSS) [15]. We characterised their muscle morphology and functional parameters and applied the European Working Group on Sarcopenia in Older People (EWGSOP) diagnostic algorithm to identify sarcopenia [2]. Inclusion and exclusion criteria and study methods have been previously described in detail [15]. The study received ethical approval from the Hertfordshire Research Ethics Committee, number 07/Q0204/68. Each participant gave written informed consent.

\section{Muscle biopsy}

Percutaneous muscle biopsies of the vastus lateralis were conducted under local anaesthetic using a Weil-Blakesley conchotome [16]. One hundred and two participants were eligible for the procedure; three were ineligible as they were taking medication that might influence subsequent wound healing $(n=2)$ or predispose to haematoma formation $(n=1)$. Biopsies from a further three participants were not suitable for analysis. Thus, the final muscle biopsy analysis sample comprised 99 participants.

\section{Immunohistochemistry}

Muscle tissue was fixed overnight at $-20{ }^{\circ} \mathrm{C}$ before being embedded in glycol methacrylate resin [17]. Serial cross-sections at $7 \mu \mathrm{m}$ were cut and stained for type II fast-twitch myofibres using the monoclonal anti-myosin fast antibody at a dilution of 1:6000 (clone MY-32; Sigma-Aldrich, Dorset, UK) (Fig. 1). Capillaries were stained by incubating separate slides with biotinylated Lectin Ulex Europeaus Agglutinin 1 (UEA-1, Vector Laboratories, Peterborough, UK) at a dilution of 1:200 for two hours (Fig. 2). Stained

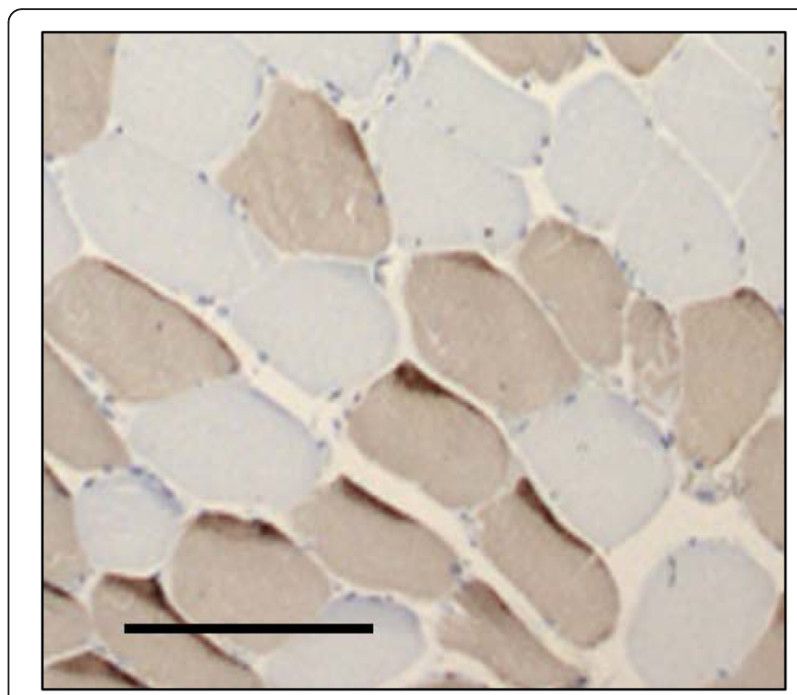

Fig. 1 A serial cross section showing differential fibre staining. Darkly stained fibres represent type II, fast fibres (anti myosin-fast antibody, clone MY32, 1:6000 Sigma- Aldrich). Bar represents $200 \mu \mathrm{M}$

sections were examined under a photomicroscope (Zeiss Axioskop II, Carl Ziess Ltd, Welwyn Garden City, UK) coupled to KS 400 image analysis software (Image Associates, Bicester, UK). Sections were viewed at a $\times 5$ magnification and digitized to obtain tissue area, myofibre number (type I, slow fibre vs type II, fast fibre) and myofibre cross-sectional areas $\left(\mu \mathrm{m}^{2}\right)$. Slow and fast fibre proportions were expressed as a percentage of total fibres. For capillaries, a digital image at a magnification of $\times 40$ was taken of the section. The total number of muscle fibres and capillaries was quantified from the whole tissue area manually from the digital image. For each section, capillary density (capillaries per $\mathrm{mm}^{2}$ ) and

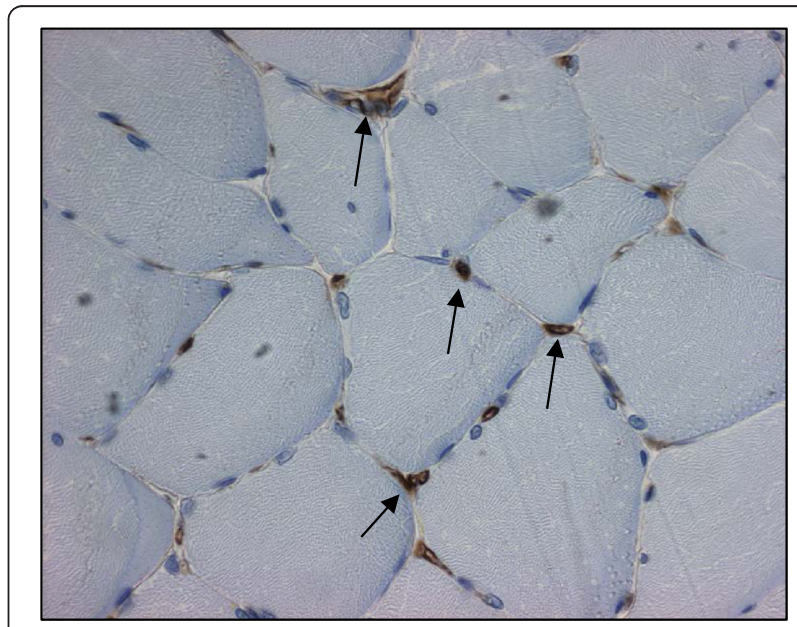

Fig. 2 A serial cross section showing capillary staining. Capillaries are stained brown and are located at the peripheries of the myocyte. (Capillaries have been stained with Ulex Europeaus Agglutinin 1, 1:200, Vector laboratories, UK, visualised at magnification $\times 40$ and are arrowed) 
capillary: fibre ratio was calculated. Satellite cells (SCs) were identified in separate tissue sections using a similar immunohistochemistry protocol with the primary antibody PAX-7 (Paired-box transcription-factor 7, Developmental Studies Hybridoma bank, University of Iowa) [18] (Fig. 3). SCs were identified by light microscopy and quantified (SC density [cells $/ \mathrm{mm}^{2}$ ] and SC to fibre ratio) using image analysis techniques as described above. Muscle morphology parameters were analysed in all samples by a blinded observer.

\section{Defining sarcopenia using the EWGSOP criteria}

For a diagnosis of sarcopenia, the EWGSOP recommend the presence of both low muscle mass and low muscle function as measured by strength and or muscle performance [2]. We use this definition based on our previous published work detailing the prevalence of sarcopenia in this cohort [5]. In our study, lean muscle mass was determined by dual-energy $\mathrm{x}$-ray absorptiometry scanning (DXA; Hologic Discovery, auto whole body software version 12.5). Isometric maximum grip strength was identified from three measurements in each hand using a standardised protocol and Jamar dynamometer [18]. A validated battery of physical performance tests was administered which included time to complete 5 chair rises and measurement of customary gait speed over $3 \mathrm{~m}$ [19]. We used values in the lowest third of the distributions of DXA derived lean mass and gait speed, and grip strength values of $<20 \mathrm{~kg}$ for women and $<30 \mathrm{~kg}$ for men, within the EWGSOP diagnostic algorithm for sarcopenia [5].

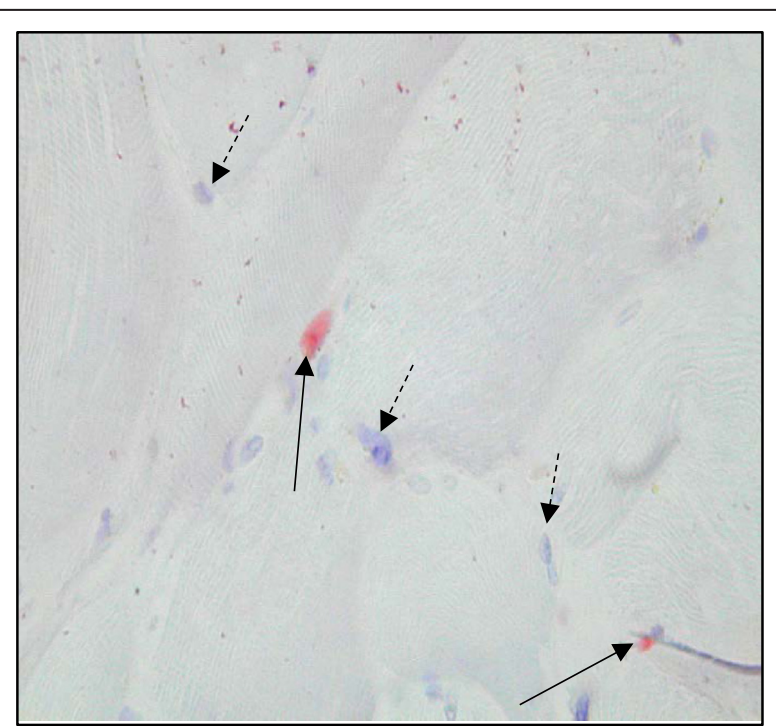

Fig. 3 A serial cross section showing satellite cells (SC). SC have stained red at the peripheries of the myocyte and are marked with arrows. Myonuclei have been counterstained with Mayer's hematoxylin and are marked with broken arrows (SC have been stained with PAX-7, Developmental Studies Hybridoma Bank, lowa, $1: 10$, magnification $\times 40$ )

\section{Statistical analysis}

Normally distributed variables were summarised using means and standard deviations (SD). Skewed variables were $\log _{\mathrm{e}}$ transformed to normal distributions as necessary; for these variables, means and SDs on the $\log _{\mathrm{e}}$ scale were back transformed to geometric means and SDs on the original scale of measurement. Student's $t$ test was used to compare age, body size, physical performance, cardiovascular fitness and fibre morphology variables between those without, and those with sarcopenia. All analyses were carried out using Stata release 13 (StataCorp, Texas, USA). A p value of $<0.05$ was considered statistically significant.

\section{Results}

\section{Participant characteristics}

Six men had sarcopenia (6.2 \% of the 97 men with complete data for items comprising the EWGSOP definition). Descriptive statistics for participant characteristics and fibre morphology in terms of satellite cell density $\left(\mathrm{cell} / \mathrm{mm}^{2}\right)$, satellite to fibre ratio type I and type II fibre distribution (\%), size/area $\left(\mu \mathrm{m}^{2}\right)$, capillary density (capillaries $/ \mathrm{mm}^{2}$ ) and capillary to fibre ratio are presented in Table 1. The total number of fibres counted to determine the capillary and satellite cell indices is also presented in Table 1.

\section{Relationships between fibre morphology and sarcopenia}

We have previously reported on the relationships between sarcopenia and age, anthropometry and muscle function [5]. Men with sarcopenia tended to have lower SC density $\left(1.7\right.$ cells $/ \mathrm{mm}^{2}$ vs 3.8 cells $\left./ \mathrm{mm}^{2}, p=0.06\right)$ and lower SC/fibre ratio (0.02 vs $0.06, p=0.06)$ than men without sarcopenia (Table 2). Men with sarcopenia also tended to have, on average, smaller slow and fast fibre areas and lower capillary to fibre ratios. However, these morphological relationships were not statistically significant (Table 2).

\section{Discussion}

We have investigated the association between skeletal muscle morphology and sarcopenia defined by the EWGSOP criteria in a population based study of community dwelling older men. In this study, there was a suggestion that the six HSS men with sarcopenia had lower average SC density and SC/fibre ratio in comparison with those without sarcopenia but the relationship was not significant at the $5 \%$ level.

Satellite cells (SC) are undifferentiated stem cells responsible for myofibre maintenance and are central to the growth and repair of muscle and have the ability to enter the cell cycle, proliferate and selfrenew [20, 21]. SC number fluctuate in younger and older adults in response to both intrinsic and 
Table 1 Participant characteristics

\begin{tabular}{|c|c|c|c|c|}
\hline & $\mathrm{N}$ & Mean (SD) & Min & Max \\
\hline Age (yrs) & 99 & $72.4(2.4)$ & 68.3 & 76.8 \\
\hline Height (cm) & 99 & $174.1(6.5)$ & 157.6 & 193.7 \\
\hline Weight (kg) & 99 & $82.7(12.6)$ & 58.1 & 118.8 \\
\hline DXA lean mass (kg) & 99 & $56.4(6.5)$ & 42.7 & 75.9 \\
\hline Grip strength (kg) & 99 & $38.7(8.1)$ & 18.0 & 66.0 \\
\hline Walking speed $(\mathrm{m} / \mathrm{s})^{a}$ & 97 & $1.1(0.2)$ & 0.5 & 1.6 \\
\hline Chair rise time $(s)^{b}$ & 96 & $17.1(4.0)$ & 8.3 & 27.2 \\
\hline Diagnosis of Sarcopenia & 97 & $6(6.2 \%)$ & & \\
\hline \multicolumn{5}{|l|}{ Fibre morphology } \\
\hline Satellite cell density $\left(\text { cell/ } \mathrm{mm}^{2}\right)^{c}$ & 69 & $3.6(2.5)$ & 0.0 & 26.4 \\
\hline Satellite cell to fibre ratio & 69 & $0.05(0.04)$ & 0.00 & 0.18 \\
\hline Fibre counts & 69 & $189.5(1.96)$ & 23.0 & 723.0 \\
\hline Slow-fibre proportion (\%) & 99 & $43.9(13.1)$ & 18.9 & 88.3 \\
\hline Fast-fibre proportion (\%) & 99 & $56.1(13.1)$ & 11.7 & 81.1 \\
\hline Slow fibre area $\left(\mu m^{2}\right)^{d}$ & 95 & $4775(1202)$ & 2059 & 9868 \\
\hline Fast fibre area $\left(\mu m^{2}\right)^{d}$ & 95 & $3953(1144)$ & 1843 & 7424 \\
\hline Capillary density (capillary/mm²) & 96 & $146.5(43.2)$ & 57.1 & 254.1 \\
\hline Capillary to fibre ratio $^{e}$ & 96 & $1.3(0.3)$ & 0.6 & 2.2 \\
\hline Fibre counts & 96 & $248.5(110.5)$ & 44.0 & 577.00 \\
\hline
\end{tabular}

${ }^{a}$ Data not available on two participants

${ }^{b}$ Data not available on three participants

'Geometric mean (SD), 30 slides were unsuitable due to suboptimal SC staining

${ }^{\mathrm{d}} 4$ slides were unsuitable for fibre area determination

$\mathrm{e}_{3}$ slides were unsuitable due to suboptimal capillary staining

extrinsic regulatory cues [22]. For example, physical activity [23] as well as with drug treatment [24]. The niche surrounding the $\mathrm{SC}$ is also a critical regulator of function and is governed by growth factors, signalling molecules as well as innervation $[22,25]$.
In support of our results, satellite cell content of fibres has been reported to decrease in the muscles of older humans. For example, Verdijk et al. showed that in older humans, SC content, specifically in type II myofibres, were lower when compared to younger controls [22]. In a study by Kadi et al. SC per fibre ratio was significantly lower in healthy older men and women compared to their younger counterparts [26]. In the later study, no inference was made on whether there was a fibre specific reduction as was reported by Verdijk et al. Therefore it appears that a reduction in satellite cells may mediate the observed muscle atrophy, specifically of type II fibres, with age. However, a detailed morphological study conducted by Purves-Smith et al. suggested that in very old individuals who may have severe muscle atrophy, both myofibre types show atrophic changes [12].

The exact reasons for a decrease in SC content or a decline in $\mathrm{SC}$ function with age are unknown. Contributing factors include alterations in the surrounding environment including denervation and oxidative damage [27] as well as decrease in activity of crucial myogenic regulatory factors coupled with an increase in negative regulators of muscle growth [28, 29]. For example, myostatin appears to suppress certain myogenic regulatory factors crucial for proliferation and differentiation and therefore has been postulated to impair function as well as self-renewal of satellite cells [30]. Taken together, it appears that loss of SC or reduced SC function diminishes the ability of ageing muscle to both hypertrophy in response to stimulus or repair and self-renew in response to injury thereby contributing to sarcopenia [27]. However, a recent study in older mice who were SC deplete suggested that neither force generation nor single fibre cross sectional area was affected by a reduction in

Table 2 Muscle morphology according to sarcopenia status among community dwelling older men

\begin{tabular}{|c|c|c|c|c|c|}
\hline & \multicolumn{2}{|c|}{ No sarcopenia } & \multicolumn{2}{|c|}{ Sarcopenia } & \multirow[t]{2}{*}{$P$ value ${ }^{* *}$} \\
\hline & $\mathrm{N}$ & Mean (SD) & $\bar{N}$ & Mean (SD) & \\
\hline \multicolumn{6}{|l|}{ Fibre morphology } \\
\hline Satellite cell density (cell/ $\left.\mathrm{mm}^{2}\right)^{a}$ & 63 & $3.8(2.4)$ & 6 & $1.7(2.2)$ & 0.061 \\
\hline Satellite cells to fibre ratio & 63 & $0.06(0.04)$ & 6 & $0.02(0.02)$ & 0.063 \\
\hline Fibre counts & 63 & $187.9(1.96)$ & 6 & $207.3(2.07)$ & - \\
\hline Slow fibre proportion (\%) & 91 & $44.3(12.4)$ & 6 & $40.8(24.0)$ & 0.530 \\
\hline Fast fibre proportion (\%) & 91 & $55.7(12.4)$ & 6 & $59.2(24.0)$ & 0.530 \\
\hline Slow fibre area $\left(\mu m^{2}\right)$ & 88 & $4820(1198)$ & 5 & $4113(1122)$ & 0.202 \\
\hline Fast fibre area $\left(\mu m^{2}\right)$ & 88 & $3960(1115)$ & 5 & $3397(1363)$ & 0.280 \\
\hline Capillary density (capillary/mm²) & 88 & $146.9(42.8)$ & 6 & $150.2(55.3)$ & 0.856 \\
\hline Capillary: fibre ratio & 88 & $1.3(0.3)$ & 6 & $1.2(0.4)$ & 0.493 \\
\hline Fibre counts & 88 & $241.9(105.0)$ & 6 & $350.8(164.0)$ & - \\
\hline
\end{tabular}

${ }^{\mathrm{a}}$ Geometric mean (SD)

** $p$ value for t-test between non sarcopenic and sarcopenic individuals as defined by the EWGSOP criteria

Sarcopenia status could not to be determined for two participants because of missing walking speed data hence maximum sample size of 97 
SC number [31]. Clearly, further studies are needed in both male and female ageing cohorts to investigate the role of SC in sarcopenia.

We observed a non-significant trend $(p>0.05)$ for men with sarcopenia to have, on average, smaller slow and fast fibre areas and lower capillary to fibre ratios. Whereas myofibre morphology measurements in relation to exercise, immobilisation and ageing have been described in a number of studies [9], this is one of the first studies to describe muscle morphology in relation to sarcopenia in community dwelling older men. Muscle mass and cross sectional area (CSA) are functions of myofibre size and number [32] and we speculate that smaller fibres seen in our study are associated with the decrease in total lean mass in men who were sarcopenic. The results of a longitudinal study by Frontera et al. [33] which revealed age related reductions in muscle cross sectional area as well as capillary to fibre ratio in men is in partial support of our cross sectional findings.

Our study had several limitations. First, the sample size was modest which will have limited statistical power. Second, the immunohistochemical methodology used to quantify the morphology parameters was open to observer error although consistent and rigorous methods were applied throughout the study in order to limit this possibility. Also, we did not evaluate SC content per specific fibre type or other indices of muscle capillarity [34]. Finally, the parameters measured may not accurately reflect the morphological changes occurring in muscle. Longitudinal studies would be helpful to more fully characterise the morphological changes that occur over time in the muscle of people with sarcopenia.

However, our study has a number of strengths. First, we have shown that it is feasible to obtain tissue from community dwelling older men in the context of an epidemiological birth cohort. The advantage of this is that morphological data can be combined with the extensive phenotypic data that has already been collected. Second, the immunohistochemical methodologies employed were based on tested protocols and can be applied to future large scale studies. For example, whereas SC have typically been studied through electron microscopy and immunofluorescence [35], few studies have employed simple immunohistochemistry techniques to quantify satellite cells in healthy individuals [26]. Our study shows that quantification of several morphological variables is possible using these methods. However, measurements of specific fibre type/SC content as well as other indices of capillarisation will need to be considered when applied to future studies.

\section{Conclusions}

Our results suggest that men with sarcopenia may have decreased satellite cell content and perhaps also smaller fibres and lower capillary to fibre ratios in comparison with men without sarcopenia. We can only speculate, given the sample size and cross sectional nature of the study, that the morphological results seen not only have an effect on muscle mass but also on muscle quality [31]. These morphological changes impact on muscle performance in men with sarcopenia, who by definition have grip strengths of less than $30 \mathrm{~kg}$, walking speed less than $0.8 \mathrm{~m}$ per second and low muscle mass [5].

The importance of this work is that the identification of morphological changes in older people with sarcopenia has the potential to identify cellular and molecular targets for therapeutic intervention. Methodological findings from this study now need to be applied to large scale studies that also include women.

\section{Competing interests}

The authors declare that they have no competing interests.

\section{Authors' contributions}

HPP, HES, AAS, CC, MW, PJS, GC participated in the conception, design and conduct of the study. LW and HES conducted statistical analyses. HPP drafted the first version of the manuscript. All authors read and approved the final manuscript.

\section{Acknowledgements}

We wish to acknowledge Katy Gould and Henry James on their contribution to this work and thank the study participants as well as the staff at the Wellcome Trust Clinical Research Facility, University Hospital Southampton for making this work possible. This study was funded by the Medical Research Council UK and the University of Southampton. The British Geriatrics Society provided additional financial support to HPP.

\section{Author details}

${ }^{1}$ Academic Geriatric Medicine, University of Southampton, University Hospital Southampton FoundationTrust (UHSFT), Tremona Road, Southampton SO16 6YD, UK. ${ }^{2}$ Medical Research Council Lifecourse Epidemiology Unit, University of Southampton, UHSFT, Tremona Road, Southampton SO16 6YD, UK.

${ }^{3}$ National Institute for Health Research Southampton Biomedical Research Centre, University of Southampton and UHSFT, Tremona Road, Southampton SO16 6YD, UK. ${ }^{4}$ Institute for Developmental Sciences, University of Southampton, UHSFT, Tremona Road, Southampton SO16 6YD, UK. ${ }^{5}$ National Institute for Health Research Collaboration for Leadership in Applied Health Research and Care: Wessex, Academic Geriatric Medicine, University of Southampton, UHSFT, Tremona Road, Southampton SO16 6YD, UK. ${ }^{6}$ National Institute for Health Research Musculoskeletal Biomedical Research Unit, University of Oxford, Oxford, UK. ${ }^{7}$ Newcastle University Institute for Ageing and Institute of Health \& Society, Newcastle University, Newcastle, UK.

Received: 16 July 2015 Accepted: 10 December 2015

Published online: 18 December 2015

\section{References}

1. Gariballa S, Alessa A. Sarcopenia: prevalence and prognostic significance in hospitalized patients. Clin Nutr. 2013;13:10.

2. Janssen I, Shepard DS, Katzmarzyk PT, Roubenoff R. The healthcare costs of sarcopenia in the United States. J Am Geriatr Soc. 2004;52(1):80-5.

3. Landi F, Cruz-Jentoft AJ, Liperoti R, Russo A, Giovannini S, Tosato M, et al. Sarcopenia and mortality risk in frail older persons aged 80 years and older: results from ilSIRENTE study. Age Ageing. 2013;42(2):203-9. doi:10.1093/ ageing/afs 194

4. Cruz-Jentoft AJ, Landi F, Schneider SM, Zuniga C, Arai H, Boirie Y, et al. Prevalence of and interventions for sarcopenia in ageing adults: a systematic review. Report of the International Sarcopenia Initiative (EWGSOP and IWGS). Age Ageing. 2014;43(6):748-59. doi:10.1093/ageing/afu115.

5. Patel HP, Syddall HE, Jameson $K$, Robinson $\mathrm{S}$, Denison $\mathrm{H}$, Roberts $\mathrm{HC}$, et al. Prevalence of sarcopenia in community-dwelling older people in the UK 
using the European Working Group on Sarcopenia in Older People (EWGSOP) definition: findings from the Hertfordshire Cohort Study (HCS). Age Ageing. 2013. Epub ahead of print.

6. Cruz-Jentoft AJ, Baeyens JP, Bauer JM, Boirie Y, Cederholm T, Landi F, et al. Sarcopenia: European consensus on definition and diagnosis: Report of the European Working Group on Sarcopenia in Older People. Age Ageing. 2010;39(4):412-23.

7. Studenski SA, Peters KW, Alley DE, Cawthon PM, McLean RR, Harris TB, et al. The FNIH sarcopenia project: rationale, study description, conference recommendations, and final estimates. J Gerontol Ser A Biol Med Sci. 2014; 69(5):547-58. doi:10.1093/gerona/glu010.

8. Chen LK, Liu LK, Woo J, Assantachai P, Auyeung TW, Bahyah KS, et al. Sarcopenia in Asia: consensus report of the Asian Working Group for Sarcopenia. J Am Med Dir Assoc. 2014;15(2):95-101. doi:10.1016/j.jamda.2013.11.025.

9. Deschenes MR. Effects of aging on muscle fibre type and size. Sports Med. 2004;34(12):809-24.

10. Aniansson A, Grimby G, Hedberg M. Compensatory muscle fiber hypertrophy in elderly men. J Appl Physiol. 1992;73(3):812-6.

11. Brunner F, Schmid A, Sheikhzadeh A, Nordin M, Yoon J, Frankel V. Effects of aging on Type II muscle fibers: a systematic review of the literature. J Aging Phys Act. 2007;15(3):336-48.

12. Purves-Smith FM, Sgarioto N, Hepple RT. Fiber typing in aging muscle. Exerc Sport Sci Rev. 2014;42(2):45-52. doi:10.1249/jes.00000000000000012

13. Degens H. Age-related skeletal muscle dysfunction: causes and mechanisms. J Musculoskelet Neuronal Interact. 2007;7(3):246-52.

14. Syddall HE, Sayer AA, Dennison EM, Martin HJ, Barker DJ, Cooper C. Cohort profile: the Hertfordshire cohort study. IntJ Epidemiol. 2005;34(6):1234-42.

15. Patel HP, Syddall HE, Martin HJ, Stewart CE, Cooper C, Sayer AA. Hertfordshire sarcopenia study: design and methods. BMC Geriatr. 2010;10:43.

16. Patel HP, Syddall HE, Martin HJ, Cooper C, Stewart C, Sayer AA. The feasibility and acceptability of muscle biopsy in epidemiological studies: findings from the Hertfordshire Sarcopenia Study (HSS). J Nutr Health Aging. 2011;15(1):10-5.

17. Britten $\mathrm{KM}$, Howarth PH, Roche WR. Immunohistochemistry on resin sections: a comparison of resin embedding techniques for small mucosal biopsies. Biotech Histochem. 1993;68(5):271-80.

18. Roberts HC, Denison HJ, Martin HJ, Patel HP, Syddall H, Cooper C, et al. A review of the measurement of grip strength in clinical and epidemiological studies: towards a standardised approach. Age Ageing. 2011;40(4):423-9.

19. Guralnik JM, Winograd CH. Physical performance measures in the assessment of older persons. Aging (Milano). 1994:6(5):303-5.

20. Hawke TJ, Garry DJ. Myogenic satellite cells: physiology to molecular biology. J Appl Physiol. 2001;91(2):534-51.

21. Tedesco FS, Dellavalle A, Diaz-Manera J, Messina G, Cossu G. Repairing skeletal muscle: regenerative potential of skeletal muscle stem cells. J Clin Invest. 2010;120(1):11-9. doi:10.1172/jci40373.

22. Verdijk LB, Dirks ML, Snijders T, Prompers JJ, Beelen M, Jonkers RA, et al. Reduced satellite cell numbers with spinal cord injury and aging in humans. Med Sci Sports Exerc. 2012;44(12):2322-30. doi:10.1249/MSS. Ob013e3182667c2e.

23. Charifi N, Kadi F, Feasson L, Denis C. Effects of endurance training on satellite cell frequency in skeletal muscle of old men. Muscle Nerve. 2003; 28(1):87-92. doi:10.1002/mus.10394.

24. Sinha-Hikim I, Cornford M, Gaytan H, Lee ML, Bhasin S. Effects of testosterone supplementation on skeletal muscle fiber hypertrophy and satellite cells in community-dwelling older men. J Clin Endocrinol Metab. 2006;91(8):3024-33.

25. Bareja A, Billin AN. Satellite cell therapy - from mice to men. Skelet Muscle. 2013;3(1):2. doi:10.1186/2044-5040-3-2

26. Kadi F, Charifi N, Denis C, Lexell J. Satellite cells and myonuclei in young and elderly women and men. Muscle Nerve. 2004;29(1):120-7. do:10.1002/mus.10510.

27. Alway SE, Myers MJ, Mohamed JS. Regulation of satellite cell function in sarcopenia. Front Aging Neurosci. 2014;6:246. doi:10.3389/fnagi.2014.00246.

28. Chakkalakal J, Brack A. Extrinsic regulation of satellite cell function and muscle regeneration capacity during aging. J Stem Cell Res Ther. 2012; (Suppl 11):001. doi:10.4172/2157-7633.s11-001.

29. Snijders $T$, Verdijk $L B$, van Loon $L$. The impact of sarcopenia and exercise training on skeletal muscle satellite cells. Ageing Res Rev. 2009;8(4):328-38. doi:10.1016/j.arr.2009.05.003.

30. McKay BR, Ogborn DI, Bellamy LM, Tarnopolsky MA, Parise G. Myostatin is associated with age-related human muscle stem cell dysfunction. FASEB J. 2012;26(6):2509-21. doi:10.1096/fj.11-198663.
31. Fry CS, Lee JD, Mula J, Kirby TJ, Jackson JR, Liu F, et al. Inducible depletion of satellite cells in adult, sedentary mice impairs muscle regenerative capacity without affecting sarcopenia. Nat Med. 2015;21(1):76-80. doi:10.1038/nm.3710.

32. Lexell J, Taylor CC. Variability in muscle fibre areas in whole human quadriceps muscle: effects of increasing age. J Anat. 1991;174:239-49.

33. Frontera WR, Hughes VA, Fielding RA, Fiatarone MA, Evans WJ, Roubenoff R. Aging of skeletal muscle: a 12-yr longitudinal study. J Appl Physiol. 2000; 88(4):1321-6.

34. Hepple RT. A new measurement of tissue capillarity: the capillary-to-fibre perimeter exchange index. Can J Appl Physiol. 1997;22(1):11-22.

35. Roth SM, Martel GF, Ivey FM, Lemmer JT, Metter EJ, Hurley BF, et al. Skeletal muscle satellite cell populations in healthy young and older men and women. Anat Rec. 2000;260(4):351-8.

\section{Submit your next manuscript to BioMed Central and we will help you at every step:}

- We accept pre-submission inquiries

- Our selector tool helps you to find the most relevant journal

- We provide round the clock customer support

- Convenient online submission

- Thorough peer review

- Inclusion in PubMed and all major indexing services

- Maximum visibility for your research

Submit your manuscript at www.biomedcentral.com/submit
Biomed Central 